\title{
Stationary solitonlike pulses in birefringent optical fibers
}

\author{
J. M. Soto-Crespo \\ Instituto de Óptica, Consejo Superior de Investigaciones Científicas, Serrano 121, 28006 Madrid, Spain \\ Nail Akhmediev and Adrian Ankiewicz \\ Optical Sciences Centre, Institute of Advanced Studies, The Australian National University, Canberra, \\ Australian Capital Territory 0200, Australia \\ (Received 22 July 1994)
}

\begin{abstract}
We study pulse propagation in birefringent fibers when differences in both phase velocities and group velocities between the two components are taken into account. We have found that both slow and fast linearly polarized solitons are unstable when the group velocity difference is high enough, and that a two-parameter family of coupled soliton states appears in this regime.
\end{abstract}

PACS number(s): 42.81.Dp, 42.81.Gs

\section{INTRODUCTION}

Propagation of solitonlike pulses in birefringent nonlinear fibers has attracted much attention in recent years [1-14]. The equations that describe pulse propagation in these fibers have been derived by Menyuk [1]. These equations are quite complicated and can be solved only in an approximate way for certain specific cases. Two main cases have been studied in depth: high and low birefringent fibers, for which two separate approximations have been developed. The case of high birefringence has been studied in detail in [1-5]. In this regime, one considers that the two linearly polarized components of the field have different phase velocities and different group velocities. Due to the nonlinearity, the pulses in these two components can capture each other, but their central frequencies become different [5] to make their group velocities equal. As a result of averaging, the fast oscillatory terms which relate the phases of the two components can be ignored and usually only trapping effects are considered in this approach [4,5].

On the other hand, the approximation of low birefringence takes into account the difference in phase velocities between the two linearly polarized components, but neglects their difference in group velocities, as this is assumed to be a higher-order effect. The two components of the soliton travel with the same group velocity and phase locking of these two components can occur. This approach has been considered numerically by Blow, Doran, and Wood [7]. In particular, polarization instabilities were first found in [7] and studied in more detail by Wright, Stegeman, and Wabnitz [9]. The full polarization dynamics of solitons in polarization-preserving fibers, in the approximation of low birefringement, has been considered in [14].

It is interesting to know what happens if both effects, viz., pulse trapping and phase locking, act together. In this paper we make a first step in trying to solve this problem. In particular, we are extending the results of [14], but now are taking into account the difference between the group velocities of the components.
Specifically, we study numerically the stationary soliton states in a birefringent fiber, considering simultaneously the differences in phase and in group velocities between the components. In doing this we are not averaging over the fast oscillatory terms, which was done in [1]. Moreover, we show that, in certain regimes of propagation, these terms play an essential role in producing stationary solutions, e.g., coupled soliton states.

Stationary solutions play an essential role in the propagation dynamics of nonlinear pulses in optical fibers. In Hamiltonian dynamical systems, they determine, to some extent, the overall dynamics of solitonlike pulses. In the low birefringence approximation, it has been shown that two different regimes of propagation of solitonlike pulses exist [14]. They are related to the linearly polarized slow and fast soliton states and also to the elliptically polarized soliton states which bifurcate from the fast soliton branch. When taking into account the different group velocities of each component, we see that even the stationary solutions become different. However, the propagation dynamics changes greatly only at quite high values of the difference in group velocities.

In this work we study numerically, and using the Poincaré sphere formalism, the stationary solitonlike solutions when polarization group velocity dispersion is taken into account. We find that when the difference between the group velocities is small, stationary solutions are similar to those in the approximation of low birefringence, i.e., they consist of slow and fast solitons. When the difference in group velocities becomes high, the slow soliton splits into two other solutions. We find that in many aspects, these solutions possess the same features as the gap solitons considered by Aceves and Wabnitz [15] and Christodoulides and Joseph [16]. In particular, the velocity of the soliton depends on the relative amplitudes of the two components.

The remainder of this paper is organized as follows. In Sec. II we formulate the problem, recalling some wellknown solutions, viz., the so-called "slow" and "fast" 
modes. In Sec. III, we study the stability of these solutions when the group velocity difference is taken into account. In Sec. IV we present and study a family of coupled soliton states. Finally, Sec. V contains our conclusions.

\section{STATEMENT OF THE PROBLEM}

Pulse propagation in a birefringent optical fiber can be described in terms of two coupled nonlinear Schrödinger equations (NLSEs). In a reference frame traveling along the $\xi$ axis with the average group velocity, this set of equations takes the form [2]

$$
\begin{aligned}
i U_{\xi}+i \delta U_{\tau}+\beta U & +\frac{1}{2} U_{\tau \tau} \\
& +\left(|U|^{2}+A|V|^{2}\right) U+(1-A) V^{2} U^{*}=0, \\
i V_{\xi}-i \delta V_{\tau}-\beta V+ & \frac{1}{2} V_{\tau \tau} \\
& +\left(A|U|^{2}+|V|^{2}\right) V+(1-A) U^{2} V^{*}=0,
\end{aligned}
$$

where $U$ and $V$ are the slowly varying envelopes of the two linearly polarized components of the field along the $x$ and $y$ axes, respectively, $\delta$ is the inverse group velocity difference, $\beta$ is half the difference between the propagation constants, $A$ is the normalized ratio of the nonlinear susceptibilities, $\xi$ is the normalized longitudinal coordinate, $\tau$ is the normalized retarded time, and the asterisk denotes complex conjugation.

The set of equations (1) has at least three integrals: the action (total energy of the pulse)

$$
Q=\int_{-\infty}^{\infty}\left(|U|^{2}+|V|^{2}\right) d \tau
$$

the momentum

$$
M=i \int_{-\infty}^{\infty}\left(U U_{\tau}^{*}-U^{*} U_{\tau}+V V_{\tau}^{*}-V^{*} V_{\tau}\right) d \tau,
$$

and the Hamiltonian

$$
\begin{aligned}
H=\int_{-\infty}^{\infty}\{ & \frac{1}{2}\left(\left|U_{\tau}\right|^{2}+\left|V_{\tau}\right|^{2}\right) \\
& -\frac{i \delta}{2}\left(U^{*} U_{\tau}-U U_{\tau}^{*}-V^{*} V_{\tau}+V V_{\tau}^{*}\right) \\
& -\beta\left(|U|^{2}-|V|^{2}\right)-\frac{1}{2}\left(|U|^{4}+|V|^{4}\right)-A|U|^{2}|V|^{2} \\
& \left.-\frac{1}{2}(1-A)\left(U^{2} V^{* 2}+U^{* 2} V^{2}\right)\right\} d \tau
\end{aligned}
$$

Equations (1) can then be written in the canonical form in terms of variational derivatives [17]

$$
i U_{\xi}=\frac{\delta H}{\delta U^{*}}, i V_{\xi}=\frac{\delta H}{\delta V^{*}} .
$$

Equations (4) and (5) define a Hamiltonian dynamical system on an infinite-dimensional phase space of two complex functions $U$ and $V$, which decrease to zero at infinity and can be analyzed on the basis of the theory of Hamiltonian systems. This means that the behavior of the solutions is defined, to a large extent, by the singular points of the system [stationary solutions of Eqs. (1)] and depends on the nature of these points (as determined by their stability). Hence the first step to make must entail finding the stationary solutions of Eqs. (1).

We are interested in pulselike solutions of (1) that are close to the soliton solutions of a single NLSE. By representing the field components in the form

$$
U=u(\xi, \tau, q) e^{i q \xi}, V=v(\xi, \tau, q) e^{i q \xi},
$$

Eqs. (1) become

$$
\begin{aligned}
i u_{\xi}+i \delta u_{\tau}-(q-\beta) u & +\frac{1}{2} u_{\tau \tau} \\
& +\left(|u|^{2}+A|v|^{2}\right) u+(1-A) v^{2} u^{*}=0 \\
i v_{\xi}-i \delta v_{\tau}-(q+\beta) v & +\frac{1}{2} v_{\tau \tau} \\
& +\left(A|u|^{2}+|v|^{2}\right) v+(1-A) u^{2} v^{*}=0,
\end{aligned}
$$

where $q$ is the soliton parameter, the soliton period depends inversely on $q$, and the energy of a soliton is proportional to $\sqrt{q}$.

Equations (7) have two simple stationary solutions, viz., linearly polarized soliton waves along the slow axis

$$
u=\frac{\sqrt{2(q-\beta)}}{\cosh [\sqrt{2(q-\beta)(\tau-\delta \xi)}]}, \quad v=0
$$

and linearly polarized soliton waves along the fast axis

$$
u=0, \quad v=\frac{\sqrt{2(q+\beta}}{\cosh [\sqrt{2(q+\beta)}(\tau+\delta \xi]} \text {. }
$$

It is remarkable that each of these solutions has zero momentum $(M=0)$, in spite of the fact that they are moving in our frame of reference. That is, these solutions show that the value of the momentum is not necessarily related to the motion of the peak amplitude of the solution.

At $\delta=0$ there is also a one-parameter family of elliptically polarized soliton states [14]. They appear at values of $q$ higher than that of the point of bifurcation $q_{\mathrm{cr}} / \beta=(3 \sqrt{33}-5) / 8 \approx 1.529, \quad Q_{\mathrm{cr}}=\sqrt{3(\sqrt{33}+1) \beta}$ $\approx 4.498 \sqrt{\beta}\left(A=\frac{2}{3}\right)$ and are analogous to the asymmetric soliton states in nonlinear directional couplers [21]. In the absence of linear birefringence $(\beta=0, \delta=0)$ and nonlinear birefringence ( $A=1)$, the pulses (8) and (9) reduce to a soliton of a single NLSE, i.e.,

$$
\sqrt{u^{2}+v^{2}}=\frac{\sqrt{2 q}}{\cosh (\sqrt{2 q} \tau)} .
$$

This solution can be linearly polarized along any direction in the $(x, y)$ plane.

Without any loss of generality, we shall take $\beta=1$ in the rest of the paper. Any other value of $\beta$ can be transformed to this one by a proper normalization of the variables. In particular, we would then have $q / \beta$ instead of $q$ after normalization, $Q / \sqrt{\beta}$ instead of $Q[14]$, and $\delta / \sqrt{\beta}$ instead of $\delta$.

\section{LINEAR STABILITY ANALYSIS}

The solutions (8) and (9) can be conveniently represented on the energy-dispersion diagram [see Fig. 1(a)]. The energy $Q$ in this diagram is given by Eq. (2). The energy 

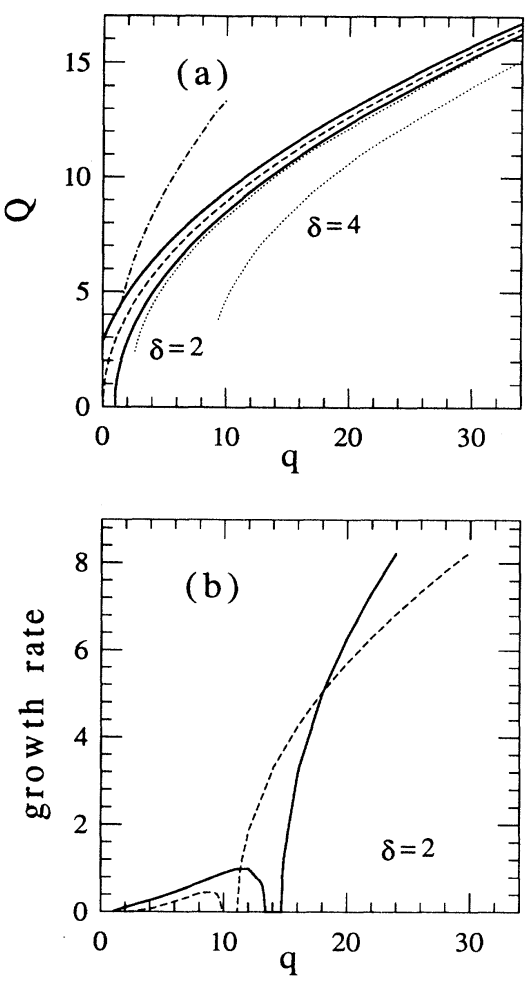

FIG. 1. (a) Energy-dispersion diagram for the fast and slow linearly polarized solitons and coupled soliton states (upper and lower solid lines, respectively). The dashed line is for a single linearly polarized soliton in the absence of birefringence. The dot-dashed line is for elliptically polarized soliton states at $\delta=0$. (b) Perturbation growth rates for the fast and slow linearly polarized solitons for $\delta=2$. The solid line shows the growth rate for the fast soliton, while dotted line is for the slow soliton.

of the NLSE soliton (10), $Q$ versus $q$, is also shown in Fig. 1(a) (by the dashed line). It is well known $[7,9,14]$ that, when $\delta=0$, the slow soliton is stable, whereas the fast soliton in unstable for most of the allowed range of $q$ [14]. The reason for this is the appearance of stationary elliptically polarized solitons above the point of bifurcation and the interaction of the soliton with radiation below the point of bifurcation.

If $\delta$ is nonzero, the stability of these two types of stationary solutions must be studied again. In fact, these states are strictly stationary solutions in certain moving frames of reference, where the stability analysis must be done. For this reason, it is convenient to make a change of coordinates to obtain a frame of reference where these solutions remain fixed:

$$
\tau=\tau^{\prime} \pm \delta \xi, \quad \xi=\xi
$$

Equations (7) then become

$$
\begin{aligned}
& i u_{\xi}+i 2 \delta u_{\tau^{\prime}}-(q-\beta) u+\frac{1}{2} u_{\tau^{\prime} \tau^{\prime}} \\
& +\left(|u|^{2}+A|v|^{2}\right) u+(1-A) v^{2} u^{*}=0 \\
& i v_{\xi}-(q+\beta) v+\frac{1}{2} v_{\tau^{\prime} \tau^{\prime}} \\
& +\left(A|u|^{2}+|v|^{2}\right) v+(1-A) u^{2} v^{*}=0
\end{aligned}
$$

or

$$
\begin{aligned}
& i u_{\xi}-(q-\beta) u+\frac{1}{2} u_{\tau^{\prime} \tau^{\prime}} \\
& +\left(|u|^{2}+A|v|^{2}\right) u+(1-A) v^{2} u^{*}=0 \\
& i v_{\xi}-i 2 \delta v_{\tau^{\prime}}-(q+\beta) v+\frac{1}{2} v_{\tau^{\prime} \tau^{\prime}} \\
& +\left(A|u|^{2}+|v|^{2}\right) v+(1-A) u^{2} v^{*}=0 .
\end{aligned}
$$

We have studied the stability of the slow and fast solitons, by using a standard technique (see [18]), based on Eqs. (12) and (13), respectively. In this way we have calculated the perturbation growth rates at the practical value of $A=\frac{2}{3}$. We have found that, for small $\delta$, namely, $\delta<1$, the results are similar to those obtained in [14] for $\delta=0$ : slow solitons are stable and fast solitons are unstable. Moreover, the fast mode is unstable in two different ranges: one of them is related to the symmetry breaking instability and the other one is related to radiative instability [14].

The new feature is that both of these ranges are shifted to higher values of $q$. In particular, the beginning of the curve for the growth rate related to the symmetry breaking instability has moved to the right in Fig. 1(b) (and now starts at $q \approx 14.8$ ). Note that the point of bifurcation of the elliptically polarized solitons from the fast modes $q=q_{\mathrm{cr}}$ is lower. This would indicate that the point of bifurcation at which the elliptically polarized solitons bifurcate from the fast modes changes from the value $q_{\mathrm{cr}} / \beta \approx 1.529$ to higher values of $q / \beta$ as $\delta$ increases.

On increasing $\delta$ further, the slow solitons also become unstable. The instability growth rate for the slow modes, as a function of $\delta$, is shown in Fig. 2 for $q=6$ (solid line), $q=10$ (dashed line), and $q=100$ (dot-dashed line). This figure illustrates that the slow mode becomes unstable only at certain threshold values of $\delta \approx 1.1$. These curves consist of two well-differentiated parts, separated by a

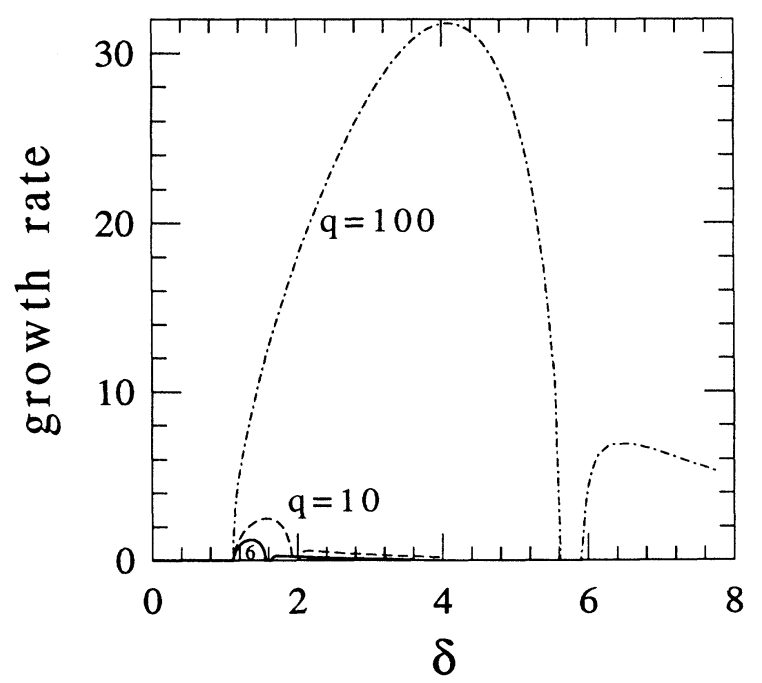

FIG. 2. Instability growth rates for the slow mode versus $\delta$ for three values of $q: q=6$ (solid line), $q=10$ (dashed line), and $q=100$ (dot-dashed line). 
small region of stability. The first part, at the lower values of $\delta$, corresponds to perturbations with real eigenvalues, while the part located on the right-hand side of the figure corresponds to complex eigenvalues. The corresponding curves for other values of $q$ follow the same tendency that these two curves indicate: when $q$ is higher the perturbation growth rates are higher and the region with real eigenperturbations is broader. It is quite remarkable that curves of this type have the same threshold value of $\delta$. This means that the slow solitons become unstable at the same critical value $\delta \approx 1.1$, independent on the values of $q$.

Figure 1(b) shows the perturbation growth rates of the slow (dashed line) and fast (continuous line) modes as a function of $q$ for $\delta=2$. As in Fig. 2, these curves consist of two segments, corresponding to zones where the perturbation with the largest growth rate has either complex (initial part) or purely real eigenvalues (for high values of $q$ ). In general, one can see that both the slow and the fast solitons have regions of instability when $\delta>\approx 1.1$. This value of $\delta$ defines the onset of instability for slow solitons. This indicates to us that other soliton states, located below the curve for slow solitons in Fig. 1(a), probably exist at values of $\delta>1$.

\section{NUMERICAL RESULTS}

The above stability results are confirmed by our extensive numerical solution of the propagation equations for many different inputs. As an example, Fig. 3(a) represents the propagation of the slow soliton with $q=100$ for $\delta=6.4$, clearly showing that it is unstable. When the slow soliton propagates a sufficient distance for the perturbation to develop, it produces a coupled soliton state plus radiation. On the other hand, Fig. 3(b) shows the propagation of the fast soliton with $q=100$ for $\delta=6.4$. Now the final state consists of a stable coupled soliton state propagating forward (having the mean group velocity) and a slow soliton which has small $q(q=2)$ and is therefore also stable (see Fig. 1). The cases shown in Fig. 3 are just two examples of many which indicate that, when $\delta>\approx 1$, coupled soliton states become stable, but linearly polarized states along the principal axes do not (the actual behavior is slightly more complicated than this, as our numerical simulations show). According to the linear stability analysis, the corresponding growth rates are 7.2 and 7.6 for the slow and the fast mode, respectively (at $q=100, \delta=6.4$ ). As the instability is seeded by a perturbation 100 times smaller than the stationary solution, this initially small perturbation becomes clearly visible at a propagation distance of $\xi \approx 0.4$ [i.e., when $0.01 \exp (7.4 \times 0.4)=0.19]$.

In the case $\delta=0$, the energy $Q$ does not depend on the velocity of the soliton. Soliton solutions that travel with a given velocity can be obtained from the solutions that are at rest in the initial frame using a Galilean transformation [10]. They have the same stability properties as solitons that do not move in the chosen frame. In the present case, Eqs. (1) are not symmetric relative to Galilean transformations. This means that the energy could depend not only on $q$, but on the group velocity of the soli- ton as well.

We have numerically propagated a great number of different input pulses. In general, after propagating a certain distance, they reach a stationary profile (see Fig 3), which moves as a unit in our frame of reference. We have analyzed these stationary pulses. They are obviously stable solutions which are stationary in a proper frame of reference. We call them "coupled soliton" states.

We have found that these coupled soliton states are, in
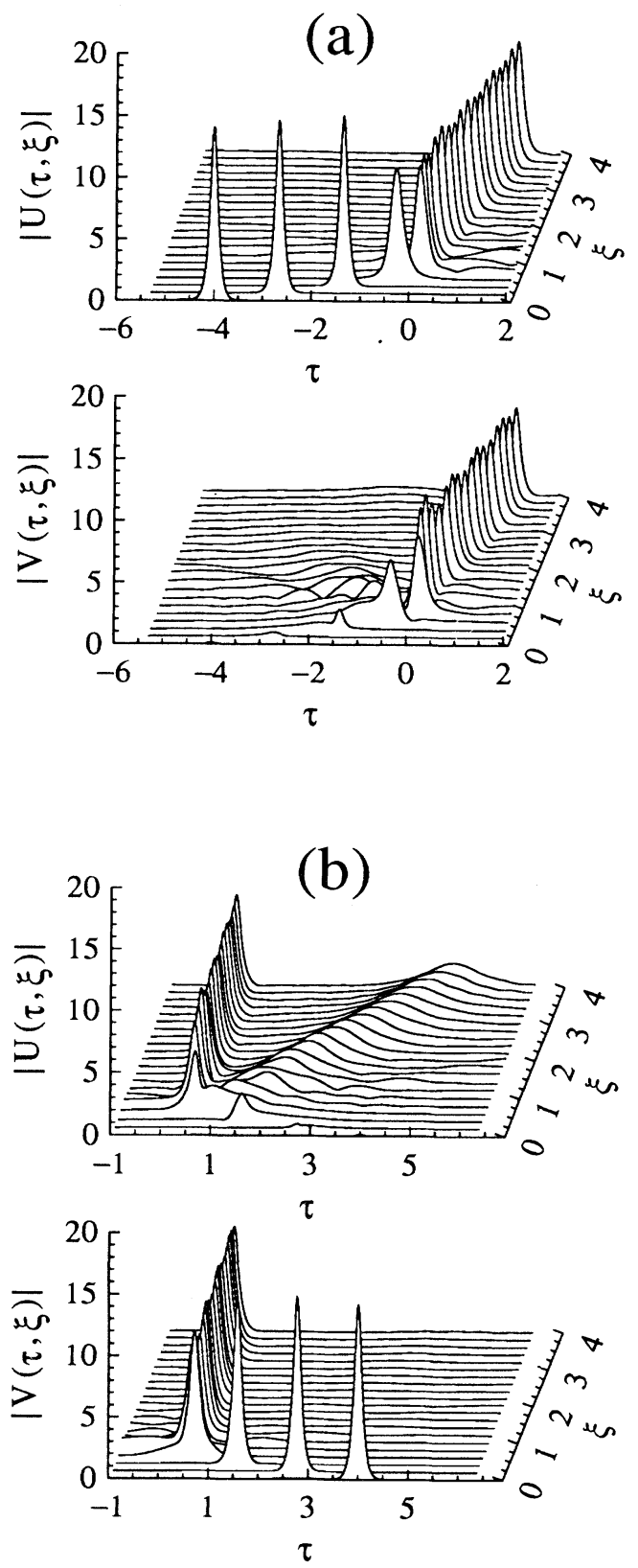

FIG. 3. Perspective plots showing the field envelopes $|U|$ and $|V|$ for propagation of (a) the slow soliton and (b) the fast soliton for $q=100, \delta=6.4$. Because both are unstable, the slow soliton transforms into a coupled soliton state radiating its excess energy, whereas the fast soliton (which has higher energy) splits into a coupled soliton state and a stable low $q(q=2)$ slow soliton. 
fact, a two-parameter family of solutions. At each $q$ they can have different values of the inverse group velocity relative to our frame of reference, $v_{\mathrm{gr}}^{-1}$, within the range $-\delta \leq v_{\mathrm{gr}}^{-1} \leq \delta$. The ratio between the two components of the soliton depends on $v_{\mathrm{gr}}^{-1}$. The corresponding $Q$ values are, however, very similar, if not identical, for the family of solutions with fixed $q$. As can be seen from Figs. 3 and 4 , the ratio of component amplitudes is not equal to unity for solitons which propagate straight $\left(v_{\mathrm{gr}}^{-1}=0\right)$. However, in the two limiting cases, when $v_{\mathrm{gr}}^{-1}$ coincides with $-\delta$ or $+\delta$ (slow and fast modes, respectively), one of the two components is zero. For a given $q$, the energy $Q$ of the coupled states could be lower than the corresponding energy of the slow soliton. We may assume that the state with the lowest energy is stable. The energy-dispersion diagram for these states, found numerically, is given in Fig. 1(a) for $\delta=2$ and 4 (dotted lines). Note that these curves are located below the curve for the slow linearly polarized soliton. As $\delta$ increases, the "coupled state" curve in this diagram moves down. It is also noteworthy that the first coupled states (those with smallest energy) that we obtain appear at those $q$ values where both fast and slow modes are unstable according to the linear stability analysis.

The peak amplitude of each component is located at the same point $t_{0}(\xi)$. The two components have the same phase at this point. They could be also $\pi$ rad out of phase because, due to the symmetry of the propagation equations, if $(U, V)$ is a solution, then $(U,-V)$ is also a solution. Hence we can conclude that the soliton is linearly polarized, at least at its peak value. The direction of polarization moves from the slow principal axis to the fast principal axis when $v_{\mathrm{gr}}^{-1}$ changes from $-\delta$ to $+\delta$.
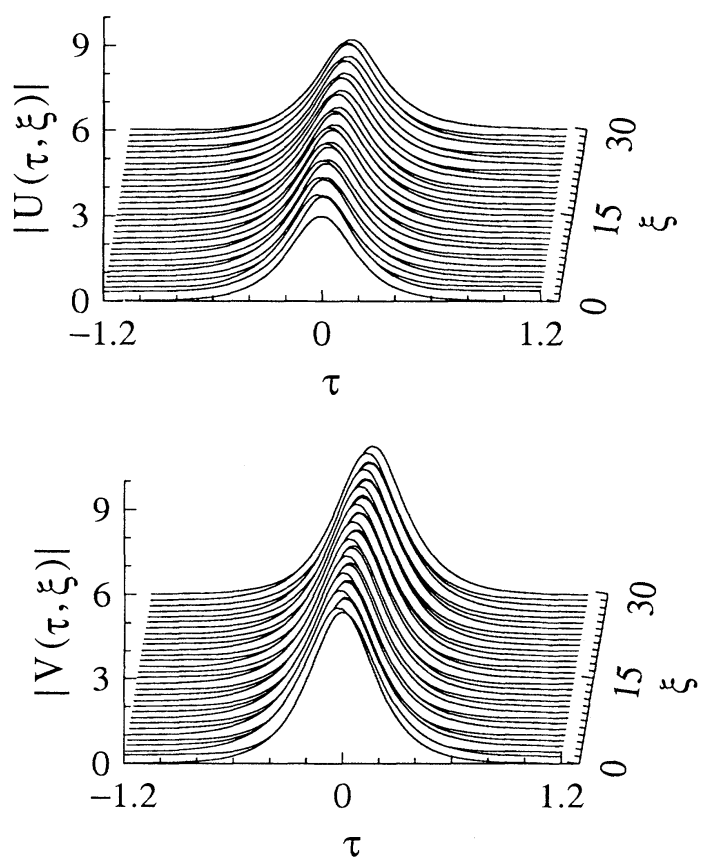

FIG. 4. Stable propagation of the coupled soliton state with $v_{\mathrm{gr}}^{-1}=0$ and $q=24.37$, for $\delta=4$.
An example of convergence to a stationary solution with $v_{\mathrm{gr}}^{-1}=0$ is given in Fig. 4. We used an input pulse with the following components:

$$
\begin{aligned}
& U=3.0 \operatorname{sech}(6.2 \tau) \exp (-i 3.11 \tau), \\
& V=5.4 \operatorname{sech}(6.2 \tau) \exp (i 3.11 \tau) .
\end{aligned}
$$

The figure shows that both components oscillate with a small amplitude around a stationary state, which therefore must be stable - it happens to have a fixed value of $q=24.37$. Note that the input amplitudes of the two components are different. The initial ratio of the components, 3.0:5.4, produces a change in the direction of propagation $\left(v_{\mathrm{gr}}^{-1} \neq 0\right)$ which is compensated for by frequency shifts of the two components which are initially \pm 3.11 .

A convenient way to analyze the polarization state of the solutions is to represent them on the Poincaré sphere $[19,20]$. In the case of solitonlike pulses, the Stokes parameters can be defined for the point of maximal amplitude of the soliton or, alternatively, as integrated Stokes parameters for the soliton as a whole. Due to results obtained in [2] (i.e., that the polarization state of the soliton is the same across the whole soliton), these two definitions give qualitatively the same results. We have to remember, however, that the two linearly polarized components of the self-trapped pulse have slightly different frequencies. This means that if phase locking occurs in addition to trapping, the relative phase difference between the two components gradually changes from zero at the center of the soliton to a nonzero value at the soliton tails. This indicates that using the definition of the Stokes vector at the center of the soliton may be more convenient.

Qualitatively, in the case $\delta=0$, the trajectories on the Poincaré sphere can be of two different types. The trajectory followed depends on whether the initial value of $q$ is higher or lower than that at the point of bifurcation. These trajectories, in the "approximation of the average profile" [14] (where we ignore radiation), are shown in Figs. 5(a) and 5(b) for cases when the energy is lower and higher than the energy at the point of bifurcation, respectively. Examples of real trajectories [taken from [14] and calculated by direct numerical integration of Eqs. (1)], including radiation effects, are shown in Figs. 6(a) and 6(b). These trajectories almost follow the trajectories of Fig. 5, but they gradually slide from one trajectory to another due to radiation. The amount of radiation depends on the proximity of the initial $q$ to the point of bifurcation. The slow soliton is represented on the sphere by the point $\mathbf{S}=\left(-S_{0}, 0,0\right)$, so that all trajectories gradually converge to this point while losing energy by emitting radiation.

We have represented the solutions of the present problem as trajectories on the Poincaré sphere. We have used the definition of Stokes parameters at the point of maximum and also integrated Stokes parameters as in [14]. In both cases the results were qualitatively equivalent. In addition, we continuously normalized the length of the Stokes vector to unity in order to represent the results on the surface of a sphere. If this was not done, the integrated Stokes vector would fall into the sphere due to the 
emission of radiation and the differential Stokes vector could move far from the surface due to oscillations of the peak amplitudes.

Figure 7 shows six trajectories of the integrated Stokes parameter for different values of $\delta$ for the input pulses:

$$
\begin{aligned}
& U=\operatorname{sech}(3.2 \tau) \exp (-i \delta \tau), \\
& V=3.3 \operatorname{sech}(3.2 \tau) \exp (i \delta \tau),
\end{aligned}
$$

where each sphere is labeled with its value of $\delta$. We can see that for low $\delta(\delta=0.4$ and 0.6$)$, the trajectories are almost the same as in the case $\delta=0$ [e.g., compare with Fig. 6(b)]. Due to radiation, the trajectories still converge to the point corresponding to the slow soliton. Hence, for small $\delta$, the dynamical situation of the solitons is qualitatively the same as in the case $\delta=0$. In fact, this range covers many cases of practical interest, when the difference in refractive indices of two components $\Delta n$ range from $10^{-6}$ to $10^{-5}$, the pulse duration is around $20-50 \mathrm{ps}$, and the group velocity dispersion is less than 1 $\mathrm{ps} /(\mathrm{nm} \mathrm{km})$. On the other hand, the value of $\delta$ can be as
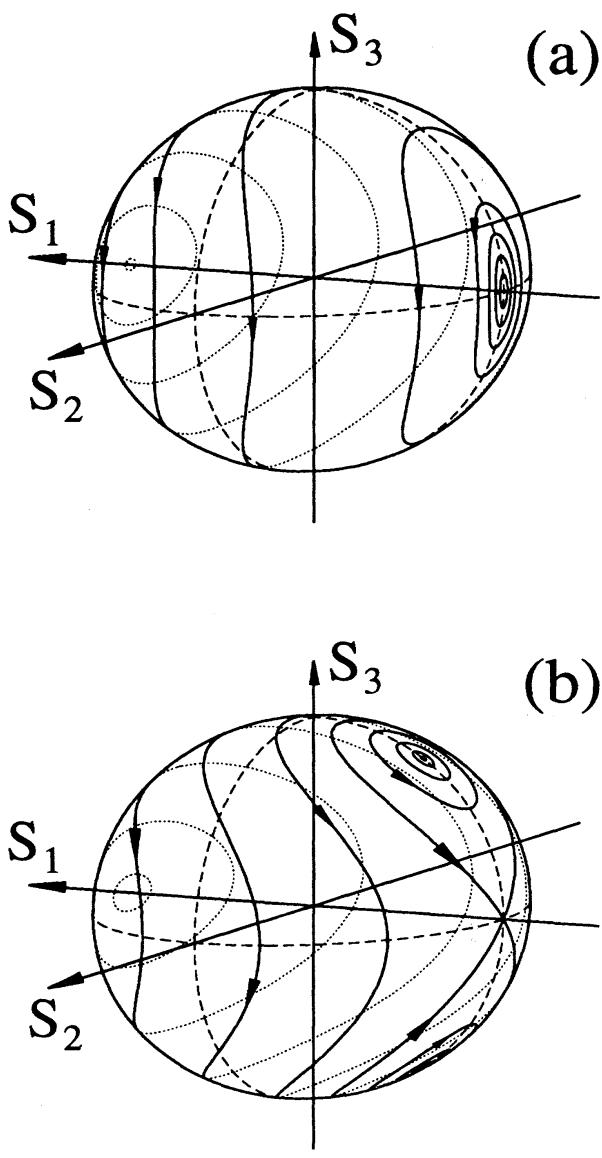

FIG. 5. Trajectories on the Poincare sphere for $\delta=0$ in the approximation of average profile [14] for (a) $q \leq q_{\mathrm{cr}}$ and (b) $q \geq q_{\mathrm{cr}}$. The value $q_{c r}$ defines the point of bifurcation of elliptically polarized solitons from the curve for fast solitons. The two stable points appearing in (b) describe elliptically polarized solitons. Their energy-dispersion curve is the dash-dotted line in Fig. 1(a).
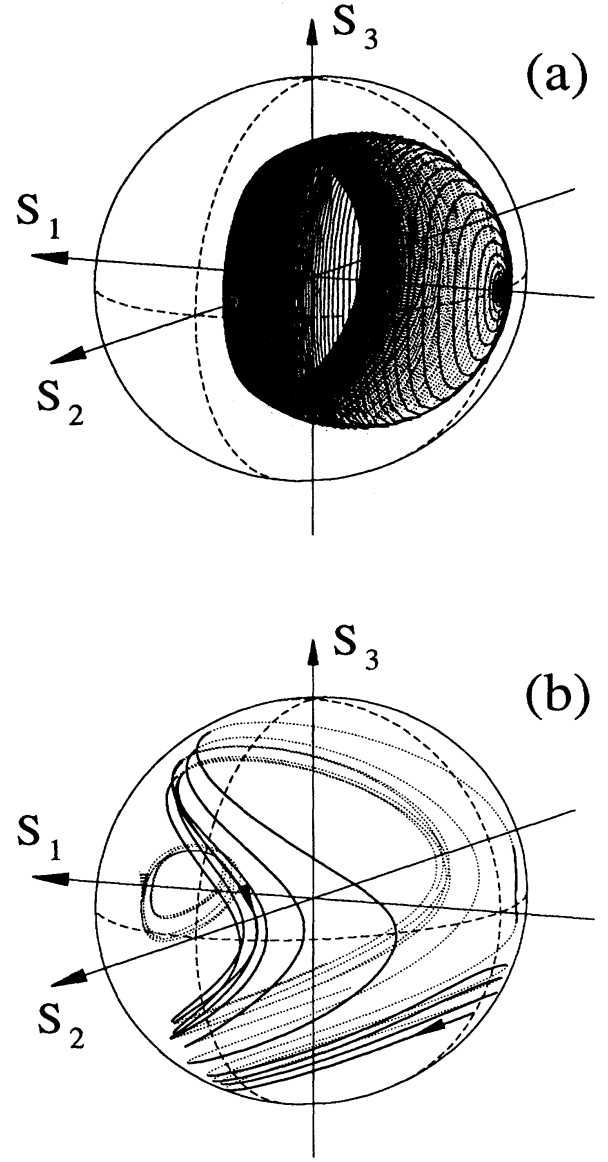

FIG. 6. Trajectories on the Poincaré sphere calculated using Eqs. (1) with $\delta=0$. Initial conditions are chosen such that (a) $q \leq q_{\mathrm{cr}}$ and (b) $q \geq q_{\mathrm{cr}}$. In (b), for clarity, only three parts of the full trajectory are shown: $0<\xi<100,400<\xi<500$, and $900<\xi<1000$.

large as 5 for pulse durations around 500 fs and $\Delta n \approx 10^{-4}$ (see [1], p. 2681). The appearance of "shadows" in some cases [4] clearly shows that the difference in group velocities can be important.

When $\delta$ increases to 0.8 , the original stationary point $\mathbf{S}=\left(S_{0}, 0,0\right)$ starts to split into three separate points: one that corresponds to the slow soliton $S=\left(S_{0}, 0,0\right)$ and two additional ones located on the equatorial line $S_{3}=0$. Figure 7 (c) shows how the trajectory loops around all three of these points. Complete splitting occurs at higher values of $\delta$. Instead of converging to the slow soliton, at these values the trajectories can converge to one of the new coupled soliton states which has two nonzero components (hence $\left|S_{1}\right|<S_{0}$ ). The convergence to one of them is clearly seen in Fig. 7(d). The other state is symmetrically located on the other side of the sphere. This is a consequence of the symmetry of the propagation equations referred to above. Note that depending on initial conditions, the trajectory can still converge to the slow soliton because it is still stable at $\delta=1.0$.

When $\delta$ is increased further, the stationary point moves along the equatorial line closer to the point defined by initial conditions (15) [Figs. 7(e) and 7(f)]. We can see 

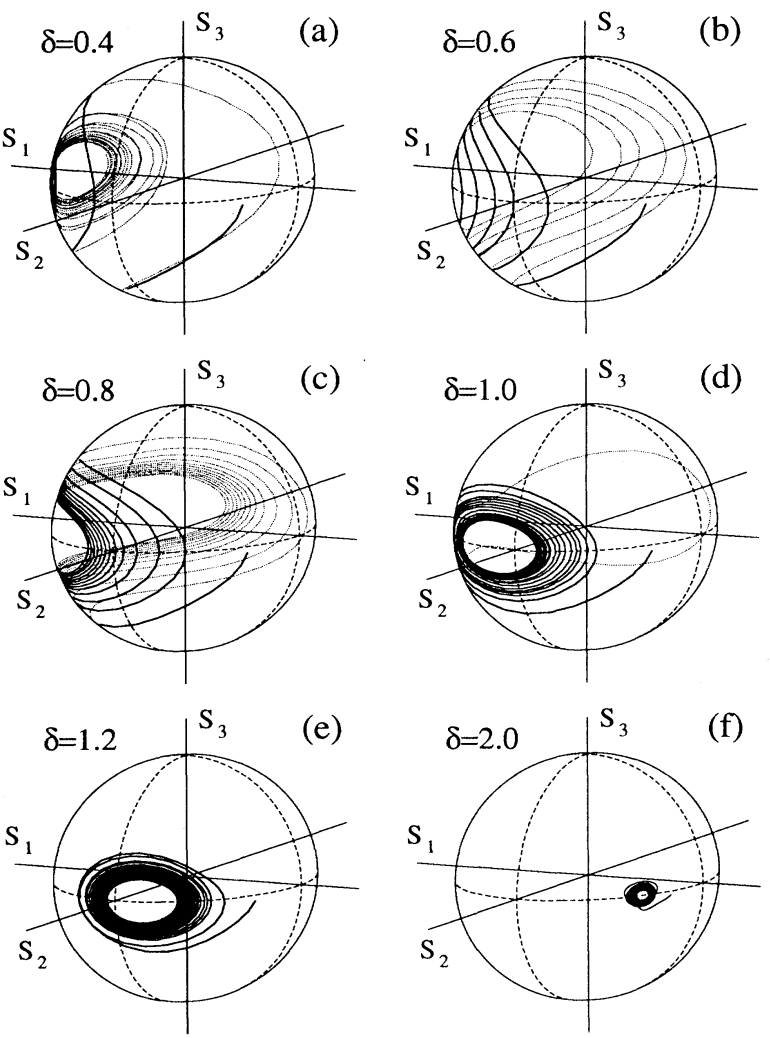

FIG. 7. Trajectories on the Poincaré sphere for similar initial conditions but different values of $\delta$. These show the appearance of two coupled soliton states as $\delta$ increases. Trajectories are calculated using integrated Stokes parameters. Up to $\delta=0.4$, the trajectories are almost the same as in the $\delta=0$ case.

that in the case of nonzero $\delta$, the stationary points are located on the equatorial line $S_{3}=0$. Each point on this line corresponds to a certain soliton state with a particular group velocity and component ratio. For given $\delta$ and fixed $q$, this constitutes a one-parameter family of solutions.

In Fig. 8 we give one more example, which shows that, at low values of $\delta$, the behavior is similar to that at $\delta=0$. The input is a fast soliton with $q=10$, which is unstable, and $\delta=0.63$. This input is gradually transformed into a slow soliton state which has the minimal energy and Hamiltonian. The Stokes parameters behave the same way as predicted by the approximation of average profile. This transformation is accompanied by radiation of small amplitude waves.

Examples of the convergence of separate trajectories to different soliton states at the same value of $\delta$, viz., $\delta=2$, but with different ratios between the components, are shown in Fig. 9. In each separate case, this ratio is related to the final value of $S_{1}$. This figure represents the trajectory of the integrated Stokes vector for various input pulses. For curve $a$ the input was

$$
\begin{aligned}
& U=2.4 \operatorname{sech}(4.2 \tau) \exp (-i 1.9 \tau), \\
& V=3.12 \operatorname{sech}(4.2 \tau) \exp (i 2.1 \tau),
\end{aligned}
$$

for curve $b$

$$
\begin{aligned}
& U=2.8 \operatorname{sech}(3.9 \tau) \exp (-i 1.9 \tau), \\
& V=2.52 \operatorname{sech}(3.9 \tau) \exp (i 2.1 \tau),
\end{aligned}
$$
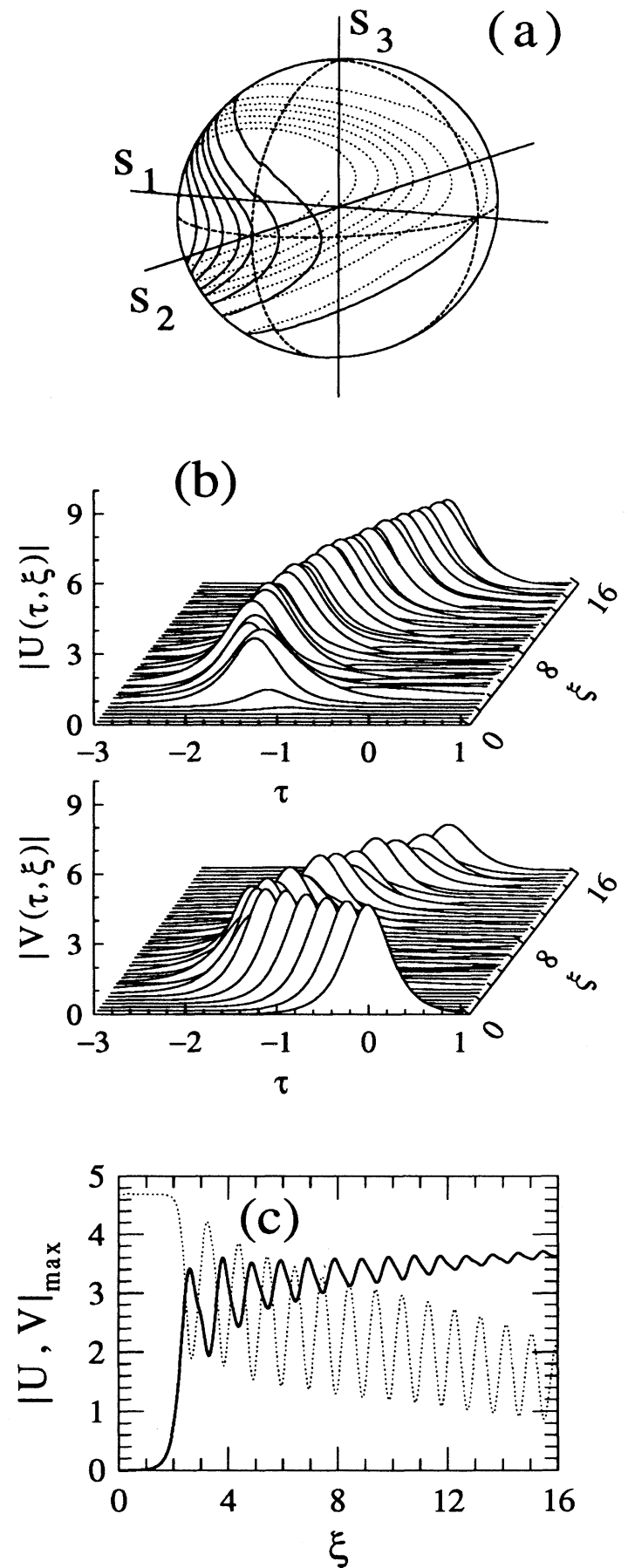

FIG. 8. (a) Trajectories on the Poincare sphere for the evolution of the fast soliton with $q=10$ at $\delta=0.63$. Due to its instability, the fast soliton gradually transforms into a slow soliton. (b) Perspective plots for the field envelopes $|U|$ and $|V|$. (c) Peak amplitudes of the two components as the pulse propagates: solid line, $|U|$; dotted line, $|V|$. This shows the decaying oscillations as the final state is approached. 


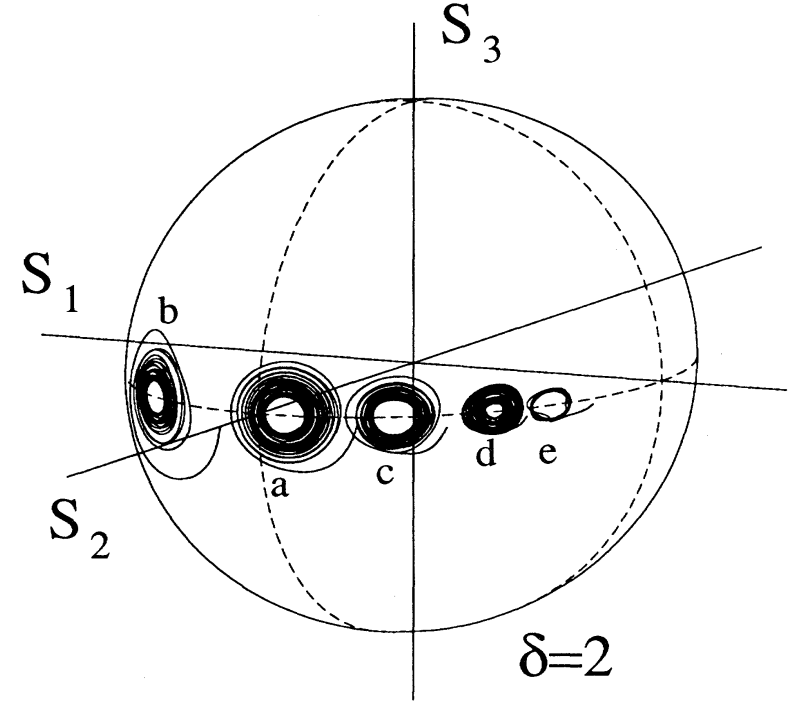

FIG. 9. Here five separate examples of the evolution of the integrated Stokes parameters for $\delta=2$ have been superimposed on the same sphere. The evolution in each trajectory is determined by its initial conditions. The solutions converge to five different coupled soliton states with similar values of $q$ and $Q$.

for curve $c$

$$
\begin{aligned}
& U=2.0 \operatorname{sech}(4.2 \tau) \exp (-i 1.9 \tau), \\
& V=3.4 \operatorname{sech}(4.2 \tau) \exp (i 2.1 \tau),
\end{aligned}
$$

for curve $d$

$$
\begin{aligned}
& U=1.5 \operatorname{sech}(4 \tau) \exp (-i 1.8 \tau), \\
& V=3.525 \operatorname{sech}(4 \tau) \exp (i 2.1 \tau),
\end{aligned}
$$

and for curve $e$

$$
\begin{aligned}
& U=\operatorname{sech}(3.2 \tau) \exp (-i 1.9 \tau), \\
& V=3.3 \operatorname{sech}(3.2 \tau) \exp (i 2.1 \tau) .
\end{aligned}
$$

In all the five cases the final states have very similar values of $q(8.0<q<8.25)$ and $Q(7.0<Q<7.3)$. The ratio of the components is different in each case and correspondingly the speeds at which they move with respect to our moving frame of reference are also different.

The value of $q$ (or $Q$ ) of the soliton state to which the solution converges is determined by the initial conditions. The initial conditions (16)-(20) are chosen in such a way that this value $q$ is close to 8 . We could choose a set of initial conditions, differing from (16)-(20), which would converge to soliton states with other $q$ values. This means that, in general, the new soliton states form a twoparameter family of solutions. Hence, generally speaking, trajectories of the solutions corresponding to the full dynamics cannot be represented on a two-dimensional surface as in [14]. Trajectories corresponding to different solutions can intersect on this surface. A higherdimensional phase space is required to classify solutions of this problem.
The soliton states for high values of $\delta$ are similar to those found by Aceves and Wabnitz [15] and Christodoulides and Joseph [16]. For each $q$, they comprise a one-parameter family of solutions, where the inverse group velocity is the parameter of the family. The ratio of the components depends on the group velocity. Although these solutions have the same qualitative properties as those found in [15], the equations that we are considering are more complicated. They include second derivative terms which are responsible for dispersion. As a result, the amplitudes of the two components are not equal at $v_{\mathrm{gr}}^{-1}=0$, while they were equal in Refs. [15] and [16]. No solutions in analytical form have been found so far because of these complications.

We should stress at this point that in this paper we have only studied stationary (phase-locked and component-trapped) solutions. The full dynamics is obviously quite an involved problem. An arbitrary initial phase is not immediately transformed into a phase-locked pulse. The full trajectory on the Poincare sphere would usually rotate around the $S_{1}$ axis. The rotation corresponds to the solutions with rotation phase [14]. This process could continue indefinitely, if we ignore radiation processes. Due to radiation, the trajectory generally slides from one closed loop to another, with decreasing Hamiltonian as the soliton loses energy on propagation.

In some cases, the trajectory can be "trapped" around some of the stationary points. A few examples are shown in Fig. 10. Initially the trajectory rotates around the $S_{1}$ axis, but eventually it may be trapped by a stationary solution located on the equatorial line $S_{3}=0$. Due to radiation, the trajectory then converges to the stationary solution. The final state, i.e., the values of $q$ (or $Q$ ) and the ratio of the components (or $S_{1}$ ), depends on the initial conditions as well as the value of $\xi$ when the trapping
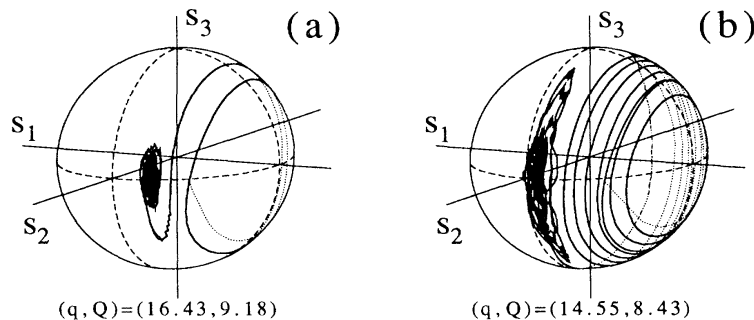

(b)
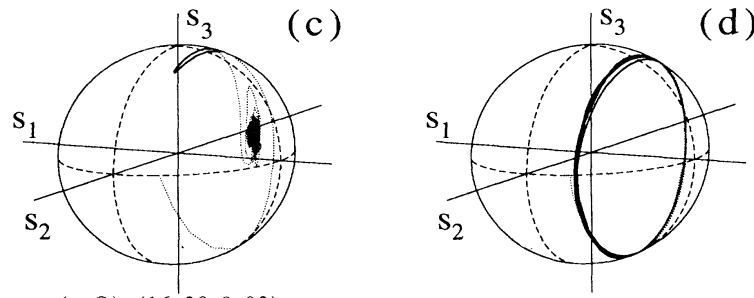

$(q, Q)=(16.30,9.03)$

FIG. 10. Trajectories on the Poincare sphere showing that some non-phase-locked solutions can be trapped and thus forced to converge to stationary solutions. 
occurs. In three of the examples of Fig. $10[(\mathrm{a})-(\mathrm{c})]$, these final values of $(q, Q)$ are shown under each example. In some cases it would take very great distances $\xi$ for the trapping to occur. An example is shown in Fig. 10(d), where the pulse still has rotating phase after rather long distances of propagation. The full dynamics of solitonlike pulses in this problem is under investigation.

\section{CONCLUSION}

In conclusion, we have found and numerically analyzed coupled soliton (phase-locked and pulse-trapped) states in birefringent optical fibers when the difference in group velocities between the two linearly polarized com- ponents is taken into account. We have shown that these soliton states form a two-parameter family of solutions. Stability properties of the new solutions have been studied. We have given examples of convergence of solitonlike pulses to these stationary solutions.

\section{ACKNOWLEDGMENTS}

The work of J.M.S.-C. was supported by the CICyT under Contract No. TIC91-0361. The work of N. A. was supported by the Australian Photonics Cooperative Research Centre. N. A. acknowledges stimulating discussions with Professor A. W. Snyder.
[1] C. R. Menyuk, IEEE J. Quantum Electron. QE-25, 2674 (1989).

[2] S. G. Evangelides, L. F. Mollenauer, J. P. Gordon, and N. S. Bergano, J. Lightwave Technol. 10, 28 (1992).

[3] H. G. Winful, Appl. Phys. Lett. 47, 213 (1985).

[4] Q. Wang, P. K. A. Wai, C.-J. Chen, and C. R. Menyuk, Opt. Lett. 16, 1388 (1991).

[5] X. D. Cao and D. D. Meyerhofer, J. Opt. Soc. Am. B 11, 380 (1994).

[6] M. N. Islam, C. D. Poole, and J. P. Gordon, Opt. Lett. 14, 1011 (1987).

[7] K. J. Blow, N. J. Doran, and D. Wood, Opt. Lett. 12, 202 (1987).

[8] C. R. Menyuk, Opt. Lett. 12, 614 (1987).

[9] E. M. Wright, G. I. Stegeman, and S. Wabnitz, Phys. Rev. A 40, 4455 (1989).

[10] R. J. Dowling, Phys. Rev. A 42, 5553 (1990).

[11] M. Romagnoli, S. Trillo, and S. Wabnitz, Opt. Quantum Electron. 24, S1237 (1992).

[12] D. F. Parker and G. K. Newbould, J. Phys. (Paris) Colloq.
50, C3-137 (1989).

[13] A. P. Mayer and D. F. Parker, Nonlinear Coherent Structures in Physics and Biology, edited by M. Remoissenet and M. Peyrard (Springer-Verlag, Berlin, 1991), p. 52.

[14] N. N. Akhmediev and J. M. Soto-Crespo, Phys. Rev. E 49, 5742 (1994).

[15] A. B. Aceves and S. Wabnitz, Phys. Lett. A 141, 37 (1989).

[16] D. N. Cristodoulides and R. K. Joseph, Opt. Lett. 16, 446 (1991).

[17] L. D. Faddeev and L. A. Takhtajan, Hamiltonian Methods in the Theory of Solitons (Springer-Verlag, Berlin, 1987).

[18] J. M. Soto-Crespo and N. N. Akhmediev, Phys. Rev. E 48, 4710 (1993).

[19] M. Born and E. Wolf, Principles of Optics (Pergamon, New York, 1959), Sec. 1.4.

[20] M. V. Tratnik and J. E. Sipe, Phys. Rev. A 35, 2965 (1987).

[21] N. N. Akhmediev and A. Ankiewicz, Phys. Rev. Lett. 70, 2395 (1993). 